\title{
Rib Fracture
}

National Cancer Institute

\section{Source}

National Cancer Institute. Rib Fracture. NCI Thesaurus. Code C34990.

A traumatic or pathologic injury to the rib in which the continuity of the rib is broken. 